Document downloaded from:

http://hdl.handle.net/10251/157296

This paper must be cited as:

Smacchia, D.; Soto Pacheco, P.; Guglielmi, M.; Morro, JV.; Boria Esbert, VE.; Raboso, D. (2019). Implementation of Waveguide Terminations With Low-Passive Intermodulation for Conducted Test Beds in Backward Configuration. IEEE Microwave and Wireless Components Letters. 29(10):659-661. https://doi.org/10.1109/LMWC.2019.2933600

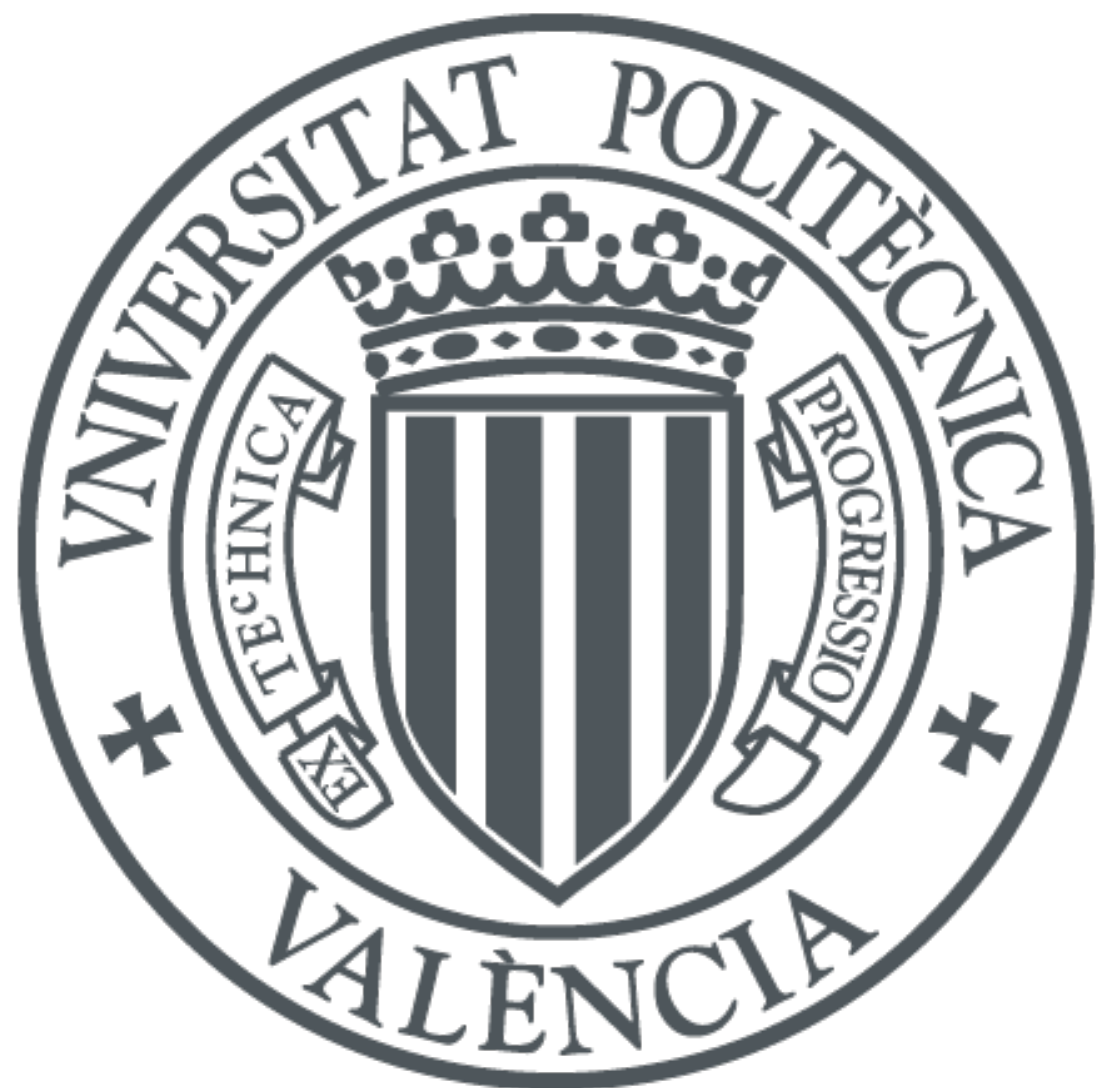

The final publication is available at

https://doi.org/10.1109/LMWC.2019.2933600

Copyright Institute of Electrical and Electronics Engineers

Additional Information

(C) 2019 IEEE. Personal use of this material is permitted. Permissíon from IEEE must be obtained for all other uses, in any current or future media, including reprinting/republishing this material for advertisíng or promotional purposes, creating new collective works, for resale or redistribution to servers or lists, or reuse of any copyrighted component of this work in other works. 


\title{
Implementation of Waveguide Terminations with Low-Passive Intermodulation for Conducted Test Beds in Backward Configuration
}

\author{
Davide Smacchia, Pablo Soto, Member, IEEE, Vicente Boria, Fellow, IEEE, and David Raboso
}

\begin{abstract}
This paper proposes a simple way to implement low-passive intermodulation (PIM) terminations in waveguide technology for test beds conceived to evaluate conducted backward PIM. By inserting a dedicated filter before the waveguide termination of the test bench, it is possible to diminish the PIM contribution from the load and consequently reduce the residual PIM noise floor of the test facility. To validate the practical application of this technique, a conducted backward low-PIM set-up working in K-band was assembled and tested. An improvement in terms of PIM performance has been obtained through the proposed solution.
\end{abstract}

Index Terms-Intermodulation distorsion, passive circuits, microwave filters, low-pass filters, PIM mitigation.

\section{INTRODUCTION}

$\mathbf{C}$ ONDUCTED low-passive intermodulation (PIM) set-ups in backward configuration are typically employed for evaluating the PIM performance of conducted elements in terms of odd PIM orders [1]-[3].

Backward PIM test beds are normally more compact than forward ones since an output diplexer is not required. In addition, they are also suitable for measuring PIM of oneport devices. However, as it has been pointed out in [4], the PIM performance of the dummy termination at the end of the common RF path is critical for backward PIM test beds. The high-power carriers reach the load, so it can generate relevant levels of passive intermodulation. Due to its particular configuration, backward test beds are completely exposed to an eventual PIM signal generated by the dummy termination. As a consequence, such termination tends to be a limiting factor in the noise floor PIM level of the test bed.

For coaxial technology this issue is overcome by manufacturing dummy terminations with long rolled cables (having longitude of hundreds of meters or even more) terminated by a coaxial load. This solution allows to gradually dissipate the

Manuscript received Month Day, Year; revised Month Day, Year; accepted Month Day, Year. This work was supported in part by the European Space Agency through several research and development contracts, in part by the Ministerio de Economa y Competitividad (Spanish Government) under Project TEC2016-75934-C4-1-R, and in part by the ESA-VSC High Power RF Space Laboratory.

D. Smacchia is with the ESA-VSC High Power RF Space Laboratory, 46022 Valencia, Spain (e-mail: davide.smacchia@val-space.com).

P. Soto and V. Boria are with the Instituto de Telecomunicaciones y Aplicaciones Multimedia, Universitat Politècnica de València, 46022 Valencia, Spain (e-mail: pabsopac@dcom.upv.es; vboria@dcom.upv.es).

D. Raboso is with the European Space Research and Technology Center, European Space Agency, 2201 AZ Noordwijk, The Netherlands (e-mail: david.raboso@esa.int).

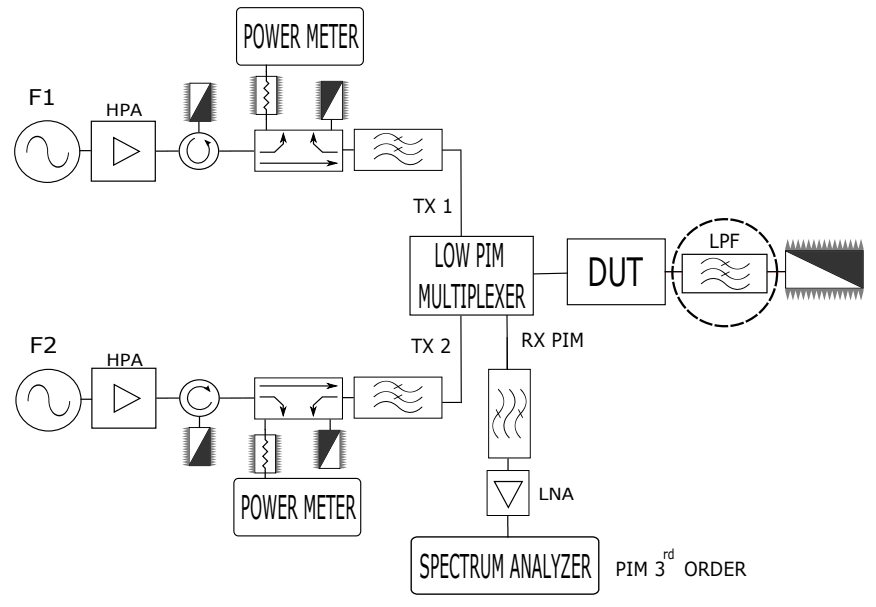

Fig. 1. Low-PIM test bench in backward configuration with the filter (circled block) placed before the waveguide dummy load.

RF power by Joule effect along the rolled cable, so the residual $\mathrm{RF}$ power arriving at the coaxial load is so attenuated that it does not add a significant PIM contribution. In addition, since coaxial cables tend to be flexible, this solution does not load the set-up with excessive mechanical tension, thus avoiding further PIM contributions in the interconnections.

In waveguide technology, however, this solution is not feasible for several reasons. First, long waveguide loads are very challenging (and expensive) to manufacture. Second, they would generate mechanical tensions to the set-up which could add residual PIM sources and even cause cracks in the structure, thus jeopardizing the integrity of the test facility.

In this paper, a solution to overwhelm this issue is proposed. It consists on inserting a suitable filter between the device under test (DUT) and the dummy termination, as shown in Fig. 1. This filter should allow the transmission carriers to go through, and at the same time reject the passive intermodulation coming from the waveguide termination, thus protecting the test bed against the PIM generated by the dummy load.

\section{Filter Considerations}

The filter to be inserted in the common RF path between the DUT and the dummy load must be designed not to affect the transmission carriers. Therefore, it must provide a passband expanding over the frequency region of the carriers. The return loss in such passband should be kept high (above $15-20 \mathrm{~dB}$ ) to avoid that a significant portion of the transmission carriers 
could travel back again, and generate unwanted resonances or additional intermodulation terms.

At the same time, the filter must have enough rejection at the frequency band where PIM signals are detected to prevent that the PIM generated by the dummy load could mask the one measured. Let be $P I M^{T}(\mathrm{dBm})$ the target PIM level that should be detected using the test bed (i.e, if the PIM level generated by a component must be certified to be below -120 $\mathrm{dBm}$, the target $P I M^{T}=-120 \mathrm{dBm}$ ). After assembling the PIM set-up with the dummy termination, a measurement of the residual PIM level of the set-up (without DUT or using a PIM-free DUT) must be carried out. Let be $P I M^{L}(\mathrm{dBm})$ the PIM noise floor measured for the test bed. The rejection the filter must provide in the PIM frequency band must satisfy:

$$
R e j .(\mathrm{dB}) \geq P I M^{L}(\mathrm{dBm})-P I M^{T}(\mathrm{dBm})+M(\mathrm{~dB})
$$

where $M$ is the design margin, which is recommended to be above $20 \mathrm{~dB}$ (to guarantee that the PIM generated by the load will always be negligible over a PIM signal of a level similar to the threshold $P I M^{T}(\mathrm{dBm})$ to be detected).

The nature of the filter to be employed depends on the frequency position of the transmission carrier band and the PIM band for the set-up. Two options are possible. For test benches where the PIM frequency band is above the transmission carriers, a low-pass filter is normally the best choice in terms of return loss, compactness and rejection. On the other hand, a high pass filter will be issued for those test benches where the PIM band is below the transmission carriers (although a combination of a band-pass filter and a high-pass filter is also possible, as shown in section III.B. of [4], if a more compact solution is sought to reduce mechanical stress). In both cases, the filter must have outstanding PIM performance. Tuning elements must therefore not be used. The filter must also be manufactured in clam-shell configuration with symmetrical width, to avoid cutting currents in the assembly [4].

It is important to mention that there can be other sources of residual PIM in the test bed. Therefore, after the insertion of the filter, the residual PIM level of the test facility could still be higher than the target one (although it will not be due to the PIM generated by the dummy termination itself).

\section{RESULTS}

The PIM mitigation technique proposed in this paper has been verified by means of a backward low-PIM set-up in Kband, on which the PIM frequency is above the transmission carriers. The key element of the set-up is a multiplexer with a transmission band between 17.89 and $20.67 \mathrm{GHz}$ and a reception band (for PIM signals) expanding from 22.7 to $24.1 \mathrm{GHz}$. The transmission band is splitted into five channels of 420 $\mathrm{MHz}$ bandwidth (and guard-bands of $170 \mathrm{MHz}$ ) implemented with low-loss high-power bandpass filters [5], [6].

Only $3^{\text {rd }}$ PIM order was evaluated, using two continuous wave $(\mathrm{CW})$ high-power input carriers of $30 \mathrm{~W}$ each. A first set of PIM measurements with only the dummy load in place was conducted. The frequencies used for each transmission carrier, as well as the frequency and level of the PIM signal measured, are compiled in Table I.
TABLE I

CONDUCTED BACKWARD PIM AT K-BAND WITH ONLY DUMMY LOAD IN PLACE, TEST SCENARIO.

\begin{tabular}{|c|c|c|c|}
\hline $\begin{array}{c}\text { Tx1 30 W CW } \\
\text { Freq. (GHz) }\end{array}$ & $\begin{array}{c}\text { Tx 2 30 W CW } \\
\text { Freq. (GHz) }\end{array}$ & $\begin{array}{c}\text { 3rd PIM } \\
\text { Freq. (GHz) }\end{array}$ & $\begin{array}{c}\text { 3rd PIM } \\
\text { Level (dBm) }\end{array}$ \\
\hline \multirow{4}{*}{17.96} & 20.34 & 22.72 & -106 \\
\cline { 2 - 4 } & 20.36 & 22.76 & -106 \\
\cline { 2 - 4 } & 20.38 & 22.80 & -106 \\
\cline { 2 - 4 } & 20.40 & 22.84 & -106 \\
\cline { 2 - 4 } & 20.42 & 22.88 & -104 \\
\cline { 2 - 4 } & 20.44 & 22.92 & -104 \\
\cline { 2 - 4 } & 20.46 & 22.96 & -104 \\
\cline { 2 - 4 } & 20.48 & 23.00 & -102 \\
\cline { 2 - 4 } & 20.50 & 23.04 & -102 \\
\hline 17.92 & 20.50 & 23.08 & -102 \\
\hline
\end{tabular}

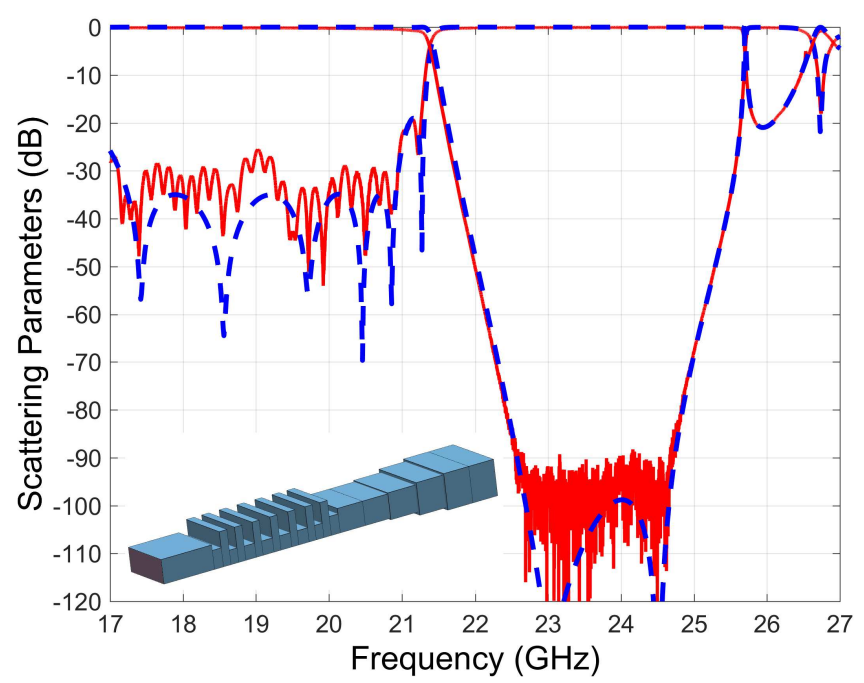

Fig. 2. Comparison between measured (continuous lines) and simulated response with FEST3D (dashed lines) of the low-pass filter used to mitigate the PIM of the dummy load in the K-band test bed.

It can be observed that the set-up was employed for several combinations of carrier frequencies, resulting in different frequencies for the PIM signal. This was done in order to check the effective PIM contribution of the test facility, getting rid of uncertainties associated to sum and destruction of several PIM contributions which could appear in backward PIM test beds (as it has been reported for the coaxial case in [7], [8]). In our case, the residual PIM level was kept approximately constant over the whole PIM frequency band of the test scenario. This result is consistent with a dominant reflected PIM source, since sum or cancellation with other PIM sources are not observed [7]. Under normal operating and assembly conditions, the load termination should be the dominant PIM source of a PIM test bed in backward configuration.

The PIM noise floor of the test bed can be assumed to be $P I M^{L}=-102 \mathrm{dBm}$ (worst case) due to the dummy load. Assuming that the weakest PIM signal to be detected is $P I M^{T}=-130 \mathrm{dBm}$, from (1), the rejection to be provided by the filter in the PIM band should be above $50 \mathrm{~dB}$.

A suitable stub-based low-pass filter (LPF) band has been chosen, since the PIM band is placed at higher frequencies than the transmission band. It has a measured rejection above $90 \mathrm{~dB}$ in the PIM band, and it is transparent to the carriers with 


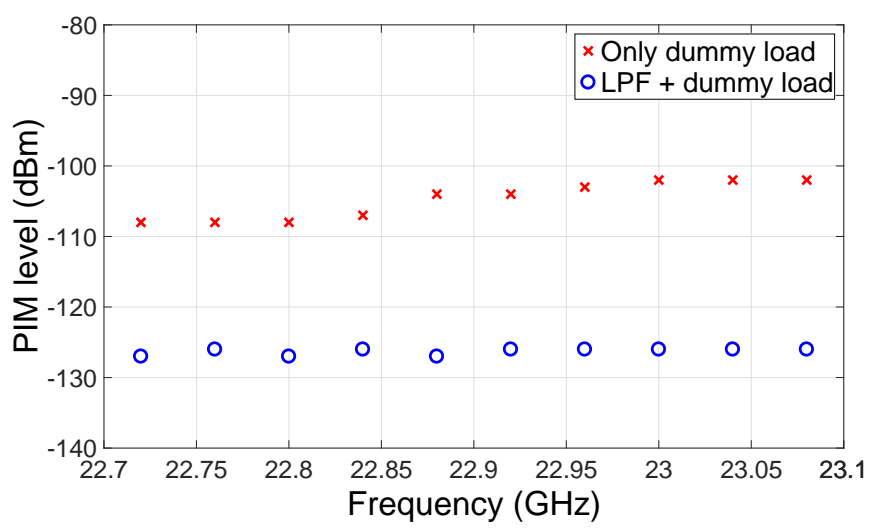

Fig. 3. PIM measurements with and without LPF before the dummy load.

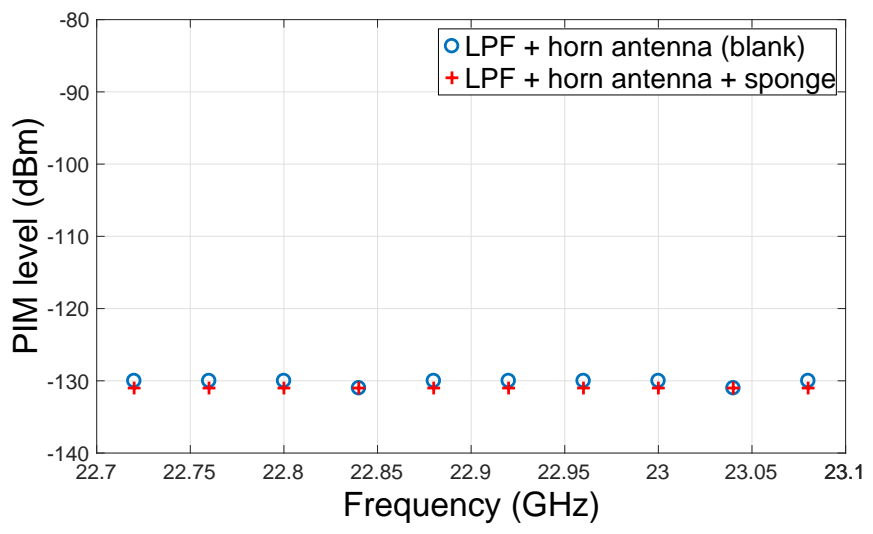

Fig. 4. PIM measurements with LPF and horn as dummy load: comparison with and without metallic sponge as PIM source.

a return loss better than $25 \mathrm{~dB}$ (see Fig. 2). The filter has an input WR42 port (since the multiplexer common port is ended in WR42), whereas the output port is WR51 (the usual one for the transmission band). The ripple measured in the return loss was due to the additional input taper that was required to perform the measurements with a network analyzer.

The low-pass filter was inserted into the test facility just before the dummy load. Figure 3 shows a comparison between the measurements conducted, with and without the LPF in place. The same frequency sets shown in Table I has been considered. As it can be observed, the insertion of the LPF provides a substantial residual PIM mitigation of the test facility, in the range between $20-25 \mathrm{~dB}$. As a result, the main PIM source of the test bed turned out to be the dummy termination, which can be blocked by the proposed low-pass filter. It must be pointed out that with the LPF in place, the PIM level is also constant over the whole frequency band. This is due to a "weaker" PIM source present in the common RF path (probably the 4-holes standard UBR220 flange used for connecting the filter input to the test bed, whose PIM performance can be improved if required by using a higher number of holes or a wider flange, according to the rule described in [9]).

In order to check the effectiveness of the $90 \mathrm{~dB}$ rejection filter to improve the PIM performance of the set-up, a second set of PIM measurements took place as follows. First, the dummy load was replaced by a horn antenna and the whole set-up was moved into an anechoic chamber. Next, a first set of measurements was conducted with the LPF connected to a horn antenna radiating towards the anechoic chamber (blank scenario). Then a metallic sponge (a typical strong PIM source) was placed at $30 \mathrm{~cm}$ from the horn antenna and a second PIM campaign took place. The results are provided in Fig. 4. The set-up showed essentially the same PIM level in both situations, thus demonstrating that the insertion of a high rejection low-pass filter blocks the PIM generated by the sponge. It is therefore a valid solution to protect a PIM test bed in backward configuration with respect to dummy loads having poor PIM performance.

\section{CONCLUSION}

This paper proposes a simple technique to reduce the residual PIM level of test benches in waveguide technology conceived to evaluate backward PIM. With the insertion of a dedicated filter before the dummy load, it is possible to reduce the residual PIM level of the test set-up related to the termination. Details for the choice of a suitable filter for this application have also been given. Several sets of measurements with a PIM set-up in K-band has been carried out to show the validity and practical use of this solution.

\section{ACKNOWLEDGMENT}

The authors are indebted with Mr. Uwe Rosenberg, from Mician Global Engineering GbR, for practical discussions related to this topic.

\section{REFERENCES}

[1] M. Bayrak and F. A. Benson, "Intermodulation products from nonlinearities in transmission lines an connectors at microwave frequencies," Proc. IEE, vol. 122, no. 4, pp. 361-367, Apr. 1975.

[2] B. Rosenberger, "The measurement of intermodulation products on passive components and transmission lines," in Proc. of 50th ARFTG Conference Digest, Dec. 1997, pp. 13-22.

[3] A. J. Christianson, J. J. Henrie, and W. J. Chappell, "Higher order intermodulation product measurement of passive components," IEEE Trans. Microw. Theory Techn., vol. 56, no. 7, pp. 1729-1736, July 2008.

[4] D. Smacchia, P. Soto, V. Boria, M. Guglielmi, C. Carceller, J. Ruiz, J. Galdeano, and D. Raboso, "Advanced compact setups for passive intermodulation measurements of satellite hardware," IEEE Trans. Microw. Theory Techn., vol. 66, no. 2, pp. 700-710, Jan. 2018.

[5] P. Soto, V. E. Boria, C. Carceller, S. Cogollos, M. Guglielmi, and D. Smacchia, "Practical design of rectangular waveguide filters with a capacitive building block providing an extra transmission zero," in Proc. of 2015 IEEE MTT-S Int. Microw. Conf., May 2015, pp. 1-4.

[6] S. Cogollos, P. Soto, V. E. Boria, M. Guglielmi, M. Brumos, B. Gimeno, and D. Raboso, "Efficient design of waveguide manifold multiplexers based on low-order EM distributed models," IEEE Trans. Microw. Theory Techn., vol. 63, no. 8, pp. 2540-2549, Aug. 2015.

[7] B. Deats and R. Hartman, "Measuring the passive-IM performance of RF cable assemblies," Microw. RF Eng., vol. 36, pp. 108-114, 1997.

[8] J. Henrie, A. Christianson, and W. J. Chappel, "Prediction of passive intermodulation from coaxial connectors in microwave networks," IEEE Trans. Microw. Theory Techn., vol. 56, no. 1, pp. 209-216, Jan. 2008.

[9] C. Vicente, D. Wolk, H. L. Hartnagel, B. Gimeno, V. E. Boria, and D. Raboso, "Experimental analysis of passive intermodulation at waveguide flange bolted connections," IEEE Trans. Microw. Theory Techn., vol. 55, no. 5, pp. 1018-1028, May 2007. 